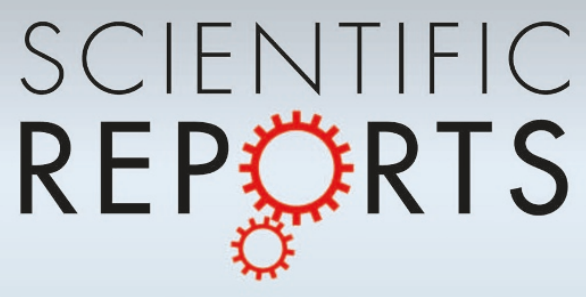

\title{
OPEN A Large-scale Cross-sectional Study of \\ Received ALK Rearrangements and EGFR Mutations in Non-small-cell Lung Cancer in Chinese Han Population
}

SUBJECT AREAS:

PREDICTIVE MARKERS

NON-SMALL-CELL LUNG CANCER

14 August 2014

Accepted

14 November 2014

Published

1 December 2014

Correspondence and requests for materials should be addressed to

L.Z. (zhangli6@mail. sysu.edu.cn)

* These authors contributed equally to this work.

Shaodong Hong 1,2,3*, Wenfeng Fang 1,2,3*, Zhihuang $\mathrm{Hu}^{1,2,3 *}$, Ting Zhou ${ }^{1,2,3}$, Yue Yan ${ }^{1,2,3}$, Tao Qin 1,2,3 Yanna Tang ${ }^{4}$, Yuxiang Ma ${ }^{1,2,3}$, Yuanyuan Zhao 1,2, Cong Xue ${ }^{1,2,3}$, Yan Huang ${ }^{1,2,3}$, Hongyun Zhao 1,2,3 \& Li Zhang ${ }^{1,2,3}$

${ }^{1}$ State Key Laboratory of Oncology in South China, Guangzhou, China, ${ }^{2}$ Collaborative Innovation Center for Cancer Medicine, Guangzhou, China, ${ }^{3}$ Department of Medical Oncology, Sun Yat-sen University Cancer Center, Guangzhou, China, ${ }^{4}$ Department of Oncology, The Fiffh Affiliated Hospital of Sun Yat-sen University, Zhuhai, China.

The predictive power of age at diagnosis and smoking history for $A L K$ rearrangements and EGFR mutations in non-small-cell lung cancer (NSCLC) remains not fully understood. In this cross-sectional study, 1160 NSCLC patients were prospectively enrolled and genotyped for $E M L 4-A L K$ rearrangements and $E G F R$ mutations. Multivariate logistic regression analysis was performed to explore the association between clinicopathological features and these two genetic aberrations. Receiver operating characteristic (ROC) curves methodology was applied to evaluate the predictive value. We showed that younger age at diagnosis was the only independent variable associated with $E M L 4-A L K$ rearrangements (odds ratio (OR) per 5 years' increment, $0.68 ; p<0.001$ ), while lower tobacco exposure (OR per 5 pack-years' increment, $0.88 ; p<0.001$ ), adenocarcinoma (OR, 6.61; $p<0.001$ ), and moderate to high differentiation (OR, 2.05; $p<0.001)$ were independently associated with EGFR mutations. Age at diagnosis was a very strong predictor of $A L K$ rearrangements but poorly predicted $E G F R$ mutations, while smoking pack-years may predict the presence of $E G F R$ mutations and $A L K$ rearrangements but with rather limited power. These findings should assist clinicians in assessing the likelihood of EML4-ALK rearrangements and EGFR mutations and understanding their biological implications in NSCLC.

$\mathrm{N}$ on-small-cell lung cancer (NSCLC) is a leading cause of cancer mortality ${ }^{1}$. Although chemotherapy remains the mainstream treatment of advanced NSCLC, small molecular tyrosine kinase inhibitors (TKIs) targeting specific driver mutations have resulted in favorable response rate, progression-free survival (PFS), and quality of life in sensitive population ${ }^{2-7}$.

Epidermal growth factor receptor (EGFR) mutations are the first discovered druggable targets in NSCLC ${ }^{8}$. Two classes of EGFR mutations, exon 19 deletions and exon 21 substitutions, account for the majority of EGFR mutations reported $(\sim 90 \%)^{9}$. These mutations are correlated with better response to gefitinib, erlotinib and afatinib $^{2-4}$. And they were more frequently observed in Asian population, never smokers, females, and patients with adenocarcinoma ${ }^{10}$. A reverse relationship between cumulative smoking pack-years and the frequency of EGFR mutations has widely been reported ${ }^{11,12}$, suggesting some predictive value of smoking status for the presence of EGFR mutations. However, the association between age at diagnosis and EGFR mutations remains controversial and little data is available regarding the predictive value of age at diagnosis on EGFR mutations $s^{10,13-15}$

Fusion of the Echinoderm microtubule-associated protein like-4 (EML4) and anaplastic lymphoma kinase $(A L K)$ represents another distinct mechanism of driver mutation in NSCLC. The fusion protein is highly oncogenic both in vitro and vivo, resulting in constitutive ALK pathway activation and ultimately cancer development ${ }^{16,17}$. Several clinical trials have demonstrated the remarkable efficacy of crizotinib for the treatment of metastatic NSCLC patients who harbor ALK rearrangements, which led to its approval from the US Food and Drug Administration and the European Medicines Agency ${ }^{6,18,19}$. EML4-ALK rearrangements were mostly reported to be associated with younger age at diagnosis and adenocarcinoma ${ }^{20-22}$. However, inconsistent reports 
still exist ${ }^{6,22,23}$. Also, the accuracy of age at diagnosis and smoking status in predicting EML4-ALK rearrangements has not been established.

One of the most challenging problems in clinical practice is to acquire adequate tumor tissue for genomic analysis. Therefore, using available clinicopathological data to predict the likelihood of certain genetic aberrations is of special significance. Furthermore, EML4$A L K$ rearrangements and EGFR mutations represent two distinct oncogenic mechanisms, which might have different clinicopathological features. However, a limited number of studies have concerned about such difference in a single dataset.

We therefore carried out this epidemiological study based on large-scale genotyped NSCLC patients to evaluate the distinct clinicopathological features associated with $A L K$ rearrangements and EGFR mutations in Chinese Han population, as well as the predictive value of age at diagnosis and smoking pack-years on these two genetic aberrations.

\section{Results}

Population characteristics. From $10^{\text {th }}$ January 2012 to $25^{\text {th }}$ April 2014, 1377 NSCLC patients were prospectively enrolled in Sun Yat-sen University Cancer Center. After excluding 102 patients who had insufficient tumor tissue for genomic analysis and another 115 patients who refused to participate in the study, a total of 1160 patients were finally included. Figure 1 outlines the process of patient selection. Overall clinicopathological features of the included patients are summarized in Table 1 . The median age at diagnosis was 57 years (range: $19-85$ years). Among them, 39.1\% were females, $54.0 \%$ were never-smokers, $78.1 \%$ had adenocarcinoma, and $43.0 \%$ were diagnosed at stage IIIB-IV. Females were more likely to be nonsmokers $(p<0.001)$. The incidence of EML4-ALK rearrangements and EGFR mutations was $8.1 \%(n=94)$ and $33.8 \%(n=392)$, respectively. Two cases of concurrent ALK rearrangement and EGFR mutation were noted, whose clinical and pathological data are presented in Supplementary Table S1 online.

Association between clinicopathological data and EML4-ALK rearrangements. Patients with $E M L 4-A L K$ rearrangements were significantly younger at diagnosis than those without such rearrangements (median age, 45 versus 58 years; $p<0.001$ ). Never smokers were more likely to harbor EML4-ALK rearrangements than smokers $(10.1 \%$ versus $6.1 \%$; $p=0.005)$. Patients with advanced NSCLC (stage IIIB-IV) had significantly higher incidence of EML4-ALK rearrangements compared with those diagnosed at stage I-IIIA $(10.6 \%$ versus $6.2 \% ; p=0.006)$. $8.9 \%$ of adenocarcinoma and $4.7 \%$ of non-adenocarcinoma had EML4$A L K$ rearrangements, respectively $(p=0.036)$. For symptoms at first onset, none of the symptoms were found to be significantly

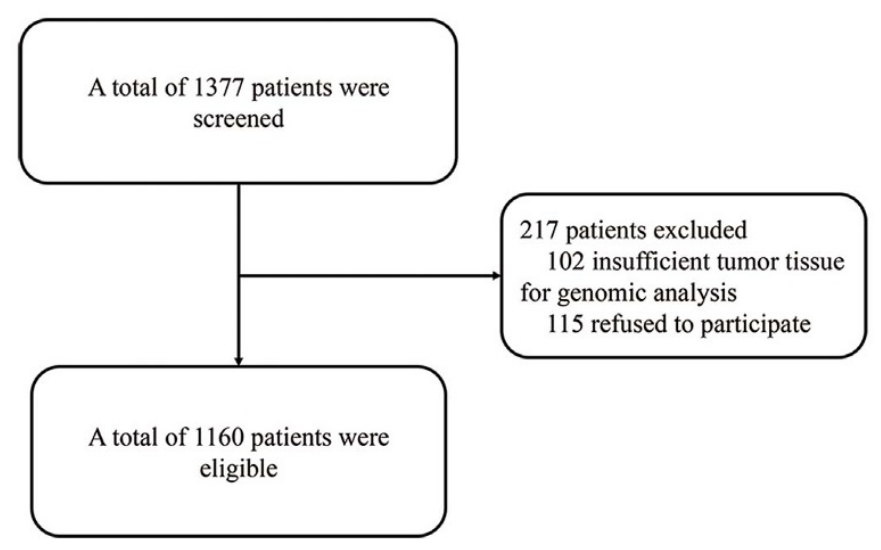

Figure 1 Flow diagram of patient selection process. associated with EGFR mutations or $A L K$ rearrangements. The results of univariate logistic analysis are shown in Table 1.

In order to adjust for confounding factors, we carried out multivariate logistic regression analysis. The results showed that only younger age at diagnosis remained independently associated with EML4-ALK rearrangements (odds ratio (OR) per 5 years' increment, $0.68 ; 95 \%$ confidence interval (CI), 0.62-0.75; $p<0.001)$. The remaining variables, including smoking status $(p=0.223)$, cancer stage $(p=0.500)$, and pathological types $(p=0.051)$ were no longer associated with EML4-ALK rearrangements.

Association between clinicopathological data and EGFR mutations. The results of univariate analysis are shown in Table 1. Female patients were more likely to have EGFR mutations compared with male patients $(46.7 \%$ versus $25.5 \%$; $p<0.001)$. Never smokers had higher incidence of EGFR mutations than smokers did (45.9\% versus $21.1 \%$; $p<0.001)$. Adenocarcinoma $(p<0.001)$ and moderate to high differentiation $(p<0.001)$ were also significantly associated with EGFR mutations. Subsequent multivariate logistic regression analysis showed that lower tobacco exposure (OR per 5 pack-years' increment, 0.88; 95\% CI, 0.85-0.92; $p<0.001$ ), adenocarcinoma (OR, 6.61; 95\% CI, 3.58-12.19; $p<0.001$ ), and moderate to high differentiation (OR, 2.05; 95\% CI, $1.55-2.71 ; p<0.001)$ were independent predictors of harboring EGFR mutations. However, gender $(p=0.154)$ and cancer stage $(p=0.767)$ were not considered to be independently associated anymore.

Age at diagnosis as a predictor of $E M L 4-A L K$ rearrangements and EGFR mutations. We sought to investigate the impacts of age at diagnosis (denoted as "age" in the following contents) on the incidence of EML4-ALK rearrangements in detail, compared with those on EGFR mutations. The incidence of EML4-ALK rearrangements decreased dramatically with increasing age, while the incidence of EGFR mutations increased with age till 50-59 years and remained nearly unchanged with age (Figure 2). Patients under the age of 30 had a $44 \%$ (7/16) incidence of EML4-ALK rearrangements, compared with a $4 \%(5 / 135)$ incidence in those above 70 years $(p<0.001)$. Notably, when stratified by gender or smoking status, similar age-distribution patterns were also observed (Figure 3).

To seek for cut-off value of age as a predictor of EML4-ALK rearrangements, ROC curve was plotted. The area under ROC curve (AUC) was $0.74(95 \% \mathrm{CI}, 0.68-0.80)$ with the cut-off age of 50.5 years (sensitivity, 73\%; specificity, 70\%) (Figure 4A). Patients under 50.5 years old had an $18.5 \%(66 / 356)$ incidence of EML4-ALK rearrangements compared with a $3.5 \%(28 / 804)$ incidence in patients above 50.5 years old $(\mathrm{OR}=6.1 ; p<0.001)$. This cut-off value also showed fair discriminative power in patients with different clinicopathological features (Table 2). In patients younger than 50.5 years old, when we added "EGFR wild type" and "adenocarcinoma" into the enrichment strategy, we got a $29.4 \%$ (58/197) incidence of EML4-ALK rearrangements.

We also plotted ROC curve for age as a predictor of EGFR mutations (Figure 4B). The AUC was only 0.52 (95\% CI, 0.49-0.56).

Smoking pack-years before diagnosis as a predictor of EML4-ALK rearrangements and EGFR mutations. The incidence of EML4-ALK rearrangements and $E G F R$ mutations by smoking pack-years was shown in Figure 5. Briefly, the incidence of EGFR mutations decreased with increasing smoking pack-years. A 5 pack-years' increment led to a $12 \%$ decrease in the likelihood of EGFR mutations. However, there was a plateau of the incidence of EGFR mutations after more than 10 pack-years of cigarettes were consumed. Even in patients with more than 80 smoking packyears, the incidence of EGFR mutations was as high as $13.2 \%$ (5/38). As for EML4-ALK rearrangements, the incidence peaked at 


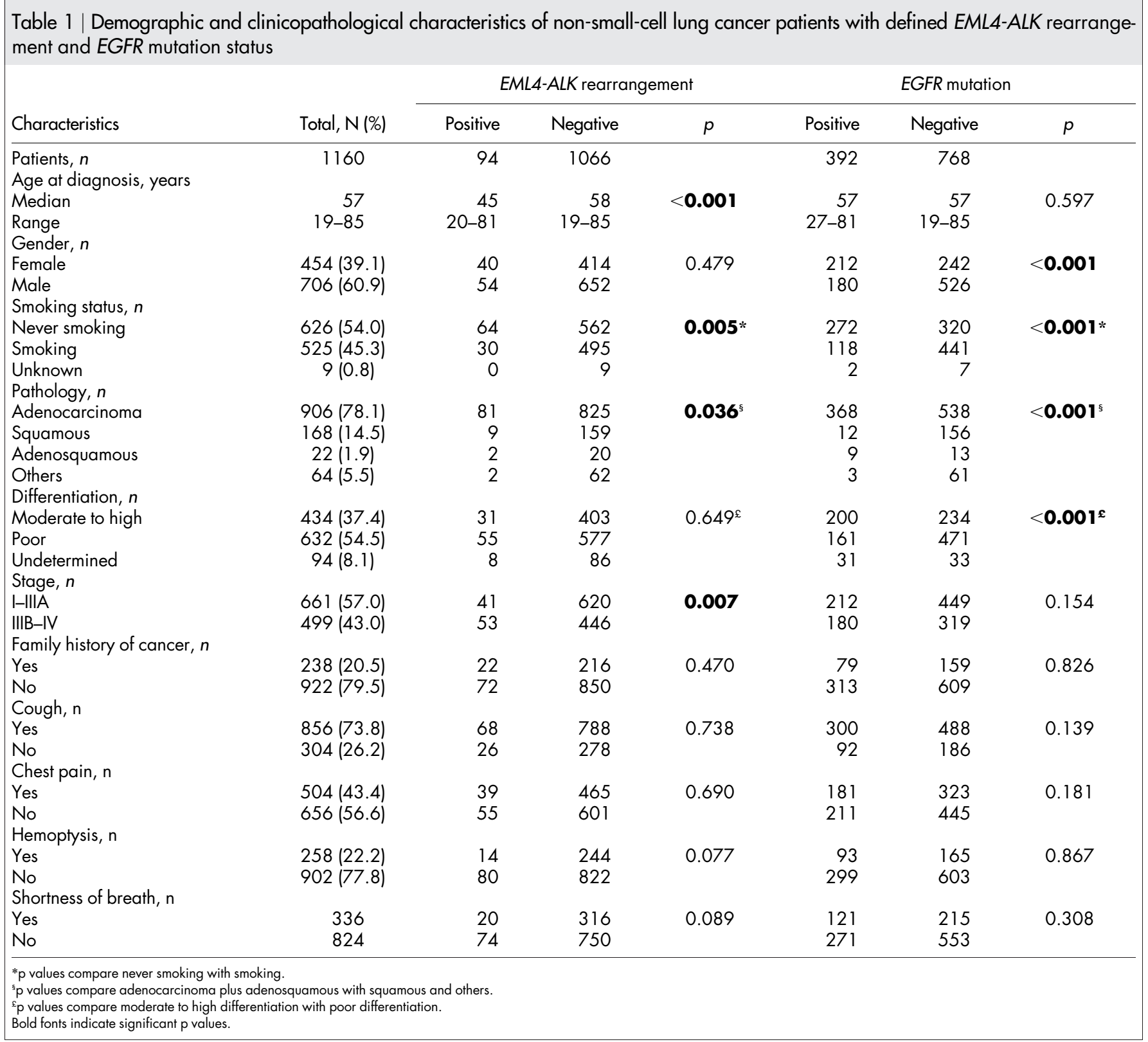

0-10 pack-years (20\%) and then dropped with increasing cigarettes smoking.

The AUC for smoking pack-years as a predictor of EML4-ALK was 0.60 (95\% CI, 0.55-0.65) with the optimal cut-off value of 10.25 packyears (sensitivity, $41 \%$; specificity, $82 \%$ ) (Figure 6A). Patients who smoked less than 10.25 pack-years were more likely to harbored EML4-ALK compared to those who smoked more than 10.25 pack-years $(11.1 \%$ versus $3.8 \%$; $p<0.001)$. For EGFR mutations, the AUC was 0.66 ( $95 \%$ CI, $0.63-0.70)$ with the cut-off value of 2.75 pack-years (sensitivity, 55\%; specificity 77\%) (Figure 6B). Patients who smoked less than 2.75 smoking pack-years had a $45.9 \%$ (291/ $634)$ incidence of EGFR mutations compared with a $17.4 \%(87 / 500)$ incidence in those who smoked more than 2.75 pack-years ( $\mathrm{OR}, 4.0$; 95\% CI, 3.0-5.3; $p<0.001$ ).

\section{Discussion}

In clinical practice, the discrimination of $A L K$ rearrangements and EGFR mutations in NSCLC has critical therapeutic implications. EGFR mutations confer sensitivity to EGFR TKIs while patients with $A L K$ rearrangements response well to ALK TKIs. However, ALK rearrangements are associated with resistance to EGFR TKIs ${ }^{21,24}$. Due to some shared features of EML4-ALK rearrangements and EGFR mutations such as adenocarcinoma histology and never/light smokers, it is important to investigate other distinct features of these two genetic aberrations. To our knowledge, this is the first study to investigate the roles of patients' clinicopathological features in predicting the presence of EML4-ALK rearrangements and EGFR mutations.

We found age at diagnosis was the only variable that independently predicted EML4-ALK rearrangements. There was an inverse relationship between age at diagnosis and the frequency of EML4$A L K$ rearrangements. A 5-year's increment in age decreased the likelihood of EML4-ALK rearrangements by $32 \%$. Using ROC curve, the cut-off age at diagnosis for predicting EML4-ALK rearrangements was determined to be 50.5 with a sensitivity of $73 \%$ and a specificity of $70 \%$. Patients younger than 50.5 years had a five-fold greater chance of harboring EML4-ALK rearrangements compared with those older than 50.5 years. Noteworthy, this cut-off value also shows fair discriminative power in patients with various clinicopathological features (Table 2). For male and female patients, the 


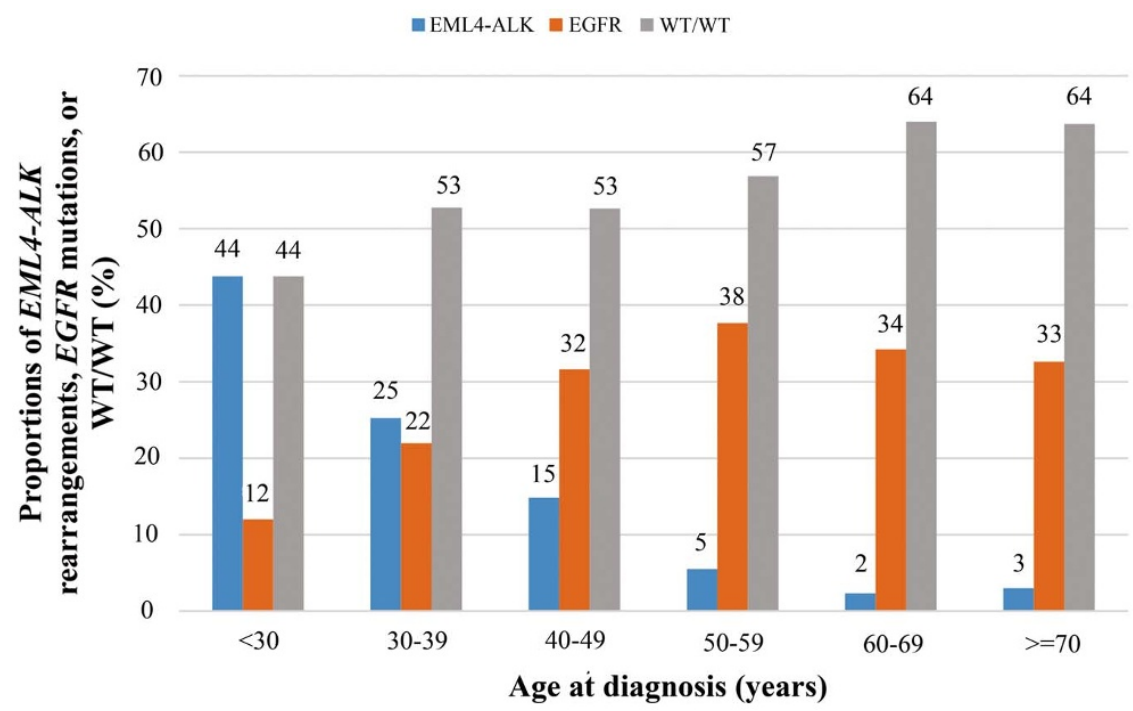

Figure $2 \mid$ The incidence of EML4-ALK rearrangements, EGFR mutations, and $W T / W T$ in non-small-cell lung cancer patients according to different age groups (at diagnosis). WT/WT, wild type $A L K$ and EGFR. There is an inverse relationship between age at diagnosis and the incidence of EML4-ALK rearrangements.

(A)

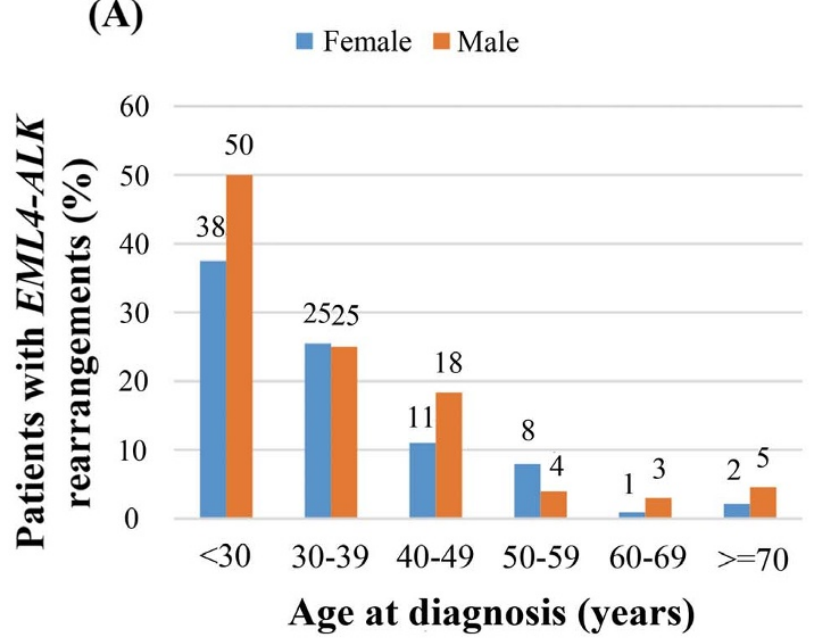

(C)

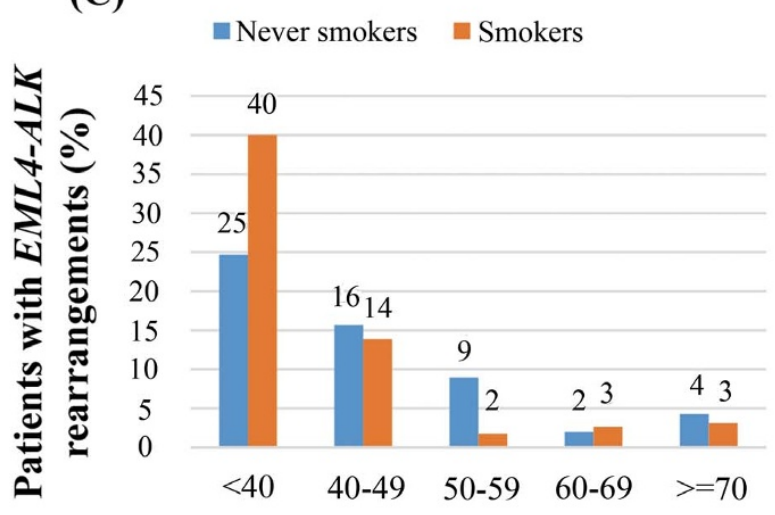

Age at diagnosis (years)
(B)

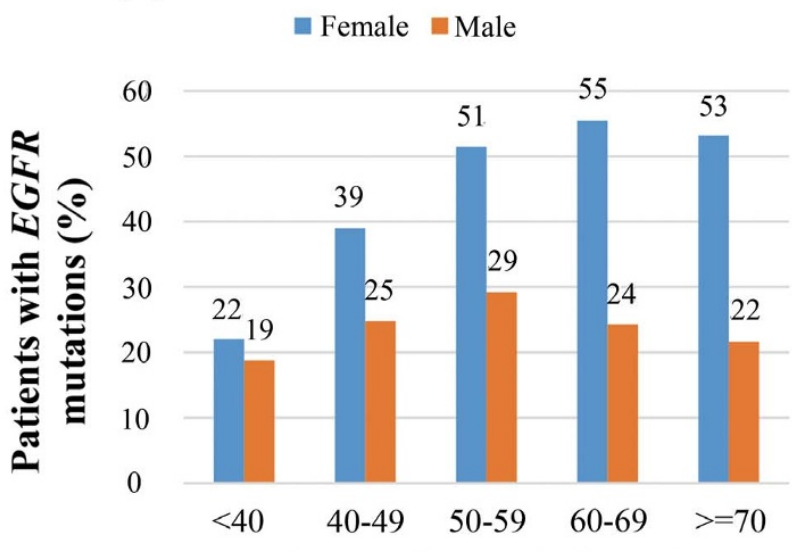

Age at diagnosis (years)

(D)

Never smokers $\quad$ Smokers

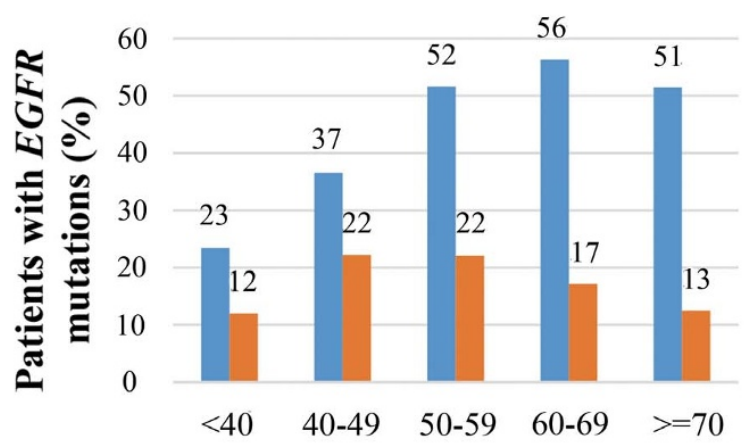

Age at diagnosis (years)

Figure $3 \mid$ Age distribution (at diagnosis) of EML4-ALK rearrangements and EGFR mutations in non-small-cell lung cancer patients at diagnosis stratified by (A) \& (B) gender and (C) \& (D) smoking status. 


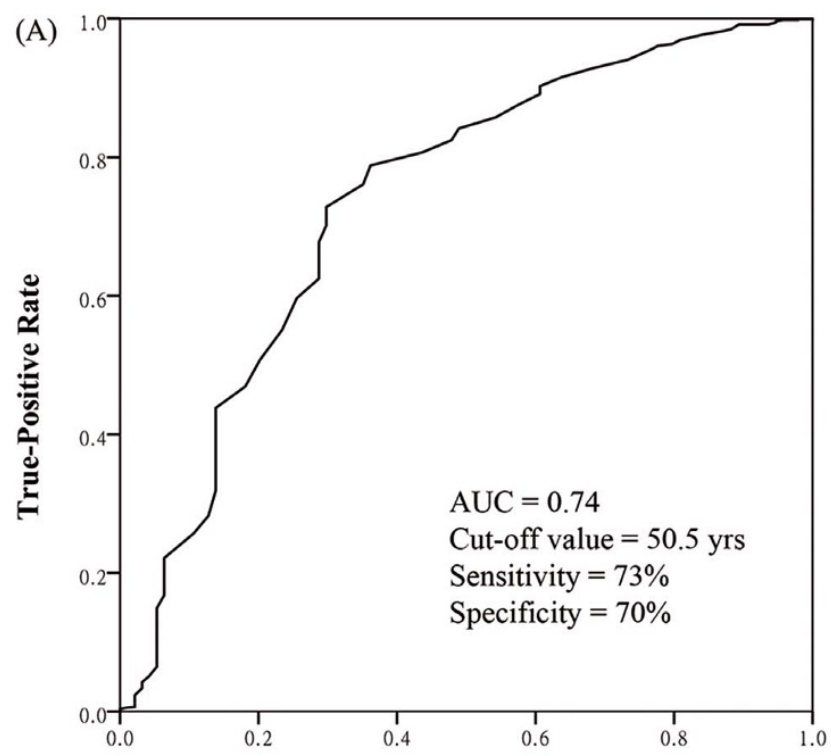

False-Positive Rate

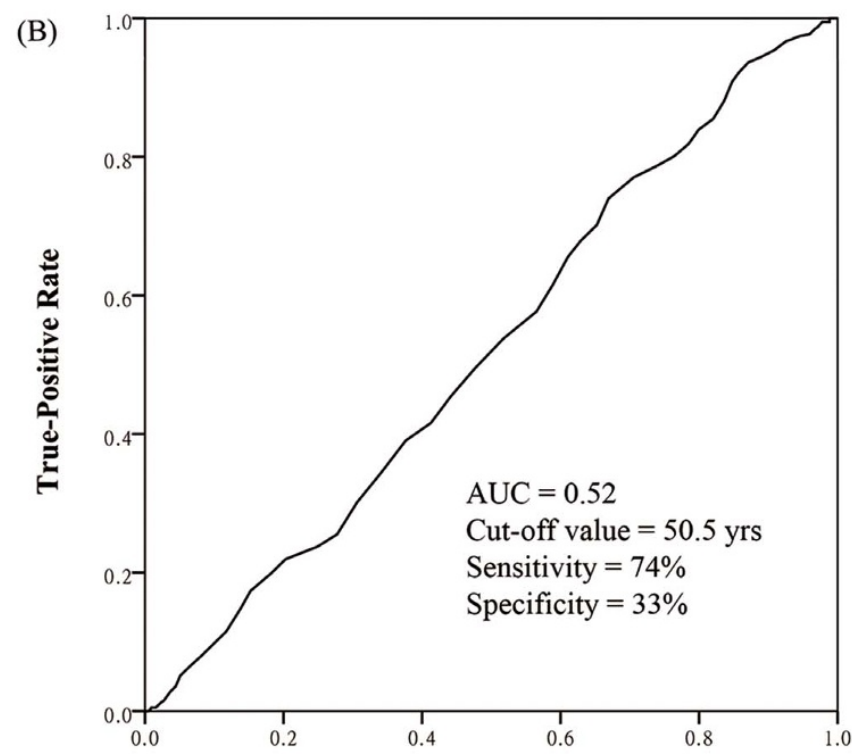

False-Positive Rate

Figure $4 \mid$ Receiver operating characteristics (ROC) curves for age at diagnosis as predictors of (A) EML4-ALK rearrangements and (B) EGFR mutations in non-small-cell lung cancer. The optimal cut-off value is the point closest the upper-left corner of the graph. AUC, areas under ROC curve.

ORs of ALK rearrangement in patients younger than 50.5 years versus those older than 50.5 years were similar $(\mathrm{OR}=6.1$ and 6.8 , respectively). This implies that gender difference in ALK rearrangement might be limited. However, in non-smokers, the discriminative power seems lower $(\mathrm{OR}=2.6)$. One possible explanation is that smoking status, though not as remarkable as age at diagnosis, still affects the incidence of ALK rearrangement. For histological subtypes (adenocarcinoma versus non-adenocarcinoma) and cancer stage (I-IIIA versus IIIB-IV), age at diagnosis also satisfactorily predict the likelihood of ALK rearrangement. Taken together, these results indicate age at diagnosis alone is a very strong predictor of $A L K$ rearrangements in NSCLC. However, attention should be paid to non-smokers older than 50.5 years old who still have $7.6 \%$ incidence of ALK rearrangement. In an enriched population (younger than 50.5 years old, EGFR wild type and with adenocarcinoma histology), we found one-third of patients harbored EML4-ALK rearrangements. This enrichment strategy is useful when assessing the likelihood of EML4-ALK rearrangements in NSCLC patients. Our results have several conflicts with a prospective $A L K$ screening study, which showed that male patients, light/never smokers and N3 stage were independently associated with $A L K$ rearrangements ${ }^{22}$. The discrepancy, which remains to be elucidated, is probably due to the small sample size in the previous study (only 16 positive cases from 116 patients), the obvious selection bias reported, or simply the ethnic difference.

Our results also revealed that the predictive power of smoking pack-years on EML4-ALK rearrangements was limited. This is unsurprising since smoking status was not an independent variable associated with EML4- $A L K$ rearrangements. Indeed, they have been sporadically reported in both smokers and nonsmokers ${ }^{22,25,26}$, indicating a lack of association between smoking status and EML4-ALK rearrangements.

As for EGFR mutations, we found that lower tobacco exposure, adenocarcinoma, and moderate to high differentiation were independently associated with EGFR mutations. Similar to a prospective EGFR screening study in Asian ${ }^{10}$, we found female was associated with $E G F R$ mutations in univariate analysis but not in multivariate logistic regression model, suggesting internal association between gender and other variables such as smoking status. Until now, it is widely believed that female rather than male patients should be tested for EGFR mutations. This unspoken prejudice may miss out a substantial of patients who will benefit from targeted therapy. A previous study which established a nomogram to predict the presence of EGFR mutations also indicated that gender has little contribution to such prediction while smoking pack-years is the strongest predictor ${ }^{27}$.

The association between age at diagnosis and EGFR mutations has long been controversial. Some studies showed that patients with EGFR mutations were older than those without EGFR mutations ${ }^{13-15}$, While other studies found no significant association ${ }^{10,28}$. In the present study, we found age at diagnosis was not associated with EGFR mutations, which was further supported by ROC curve (AUC = 0.52 ). These results suggest that the likelihood of EGFR mutations is poorly predicted by age at diagnosis. One possible explanation might be that there is a peak incidence of EGFR mutations around 60 years old (Figure 1, "n" shape distribution). This was also supported by the nomogram model from Girard et al's study which shows that patients aged between 60 and 70 have higher probability of EGFR mutations ${ }^{27}$.

Finally, we explored the roles of smoking pack-years in predicting EGFR mutations. We found the incidence of EGFR mutations was inversely related to smoking pack-years, similar to a previous study ${ }^{11}$. In that study, they concluded that smoking pack-years strongly predicted $E G F R$ mutations $(A U C=0.78)$. No patients that have smoked more than 75 pack-years harbor EGFR mutations. In the current study, however, the AUC was lower (AUC = 0.66). Indeed, we observed a plateau of the incidence of EGFR mutations after more than 10 pack-years of cigarettes consumption, suggesting that smoking has no cumulative effects on EGFR mutations. Patients who have smoked more than 80 pack-years still had a $13.2 \%$ incidence of EGFR mutations. Therefore heavier smokers should still be considered for EGFR mutation tests ${ }^{29}$. This finding also explains why the predictive accuracy of smoking pack-years reported here was lower than previously believed ${ }^{11}$.

The distinct age-distribution patterns of EML4-ALK rearrangements and EGFR mutations may also imply the difference of oncogenic potency. NSCLCs with EGFR mutations are generally dormant and would take a longer time to become clinically detectable, resulting in accumulated occurrence of EGFR mutant tumors in patients of 
Table 2 | Odds ratio of EML4-ALK rearrangements in patients younger than 50.5 yrs versus patients older than 50.5 yrs, stratified by clinicopathological features

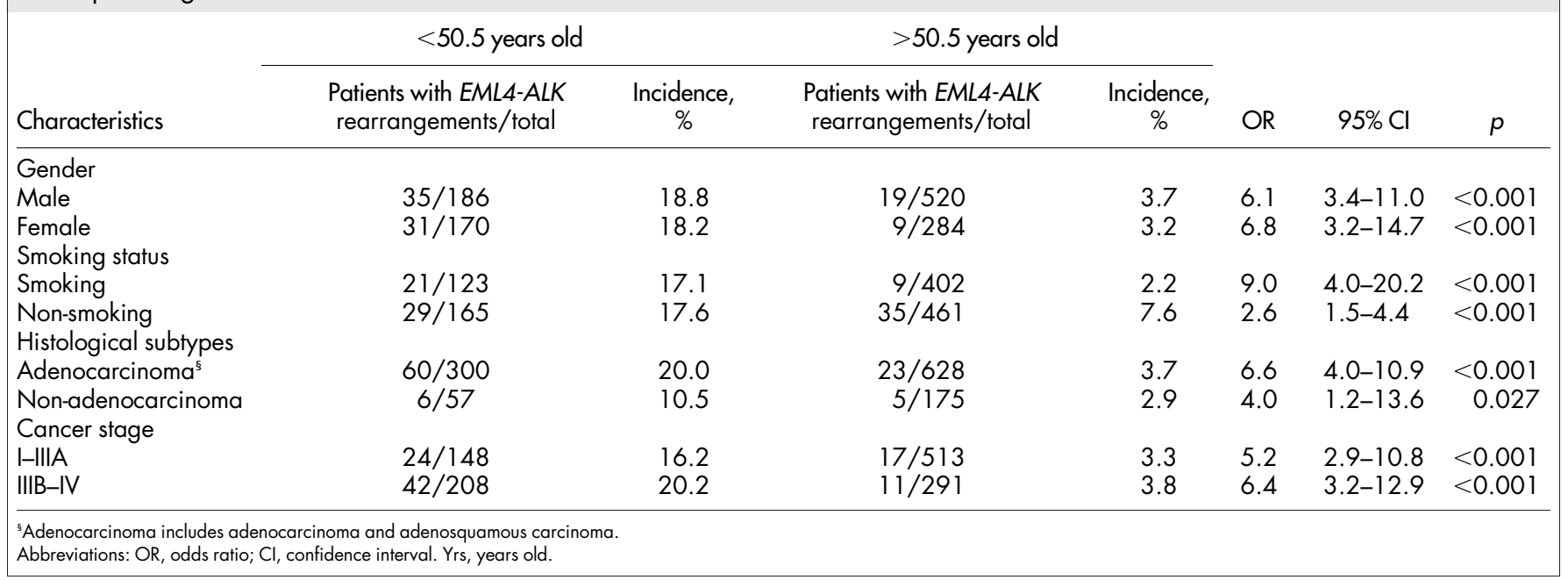

relatively older age. While chromosomal abnormalities may result in structural changes of critical proteins and hence more aggressive tumors which require less time to become overt diseases. In favor of this point, other cancer types including anaplastic large cell lymphomas, inflammatory myofibroblastic tumor, and neuroblastoma that harbor $A L K$ rearrangements occur predominantly in children and young adults. Other fusion genes in NSCLC, such as ROS1 and $R E T$ are also associated with younger age at diagnosis ${ }^{30,31}$, suggesting a class-specific characteristic of fusion genes that differ from point mutations or indels (insertions and deletions) such as Kras mutations and PI3CA mutations ${ }^{32,33}$. Therefore, in very young NSCLC patients, the tests of $A L K$ rearrangements should be given priority over EGFR mutations (especially in those younger than 30 years old). Furthermore, in vitro and in vivo studies to investigate the biological difference between these two oncogenic mechanisms are warranted.
Due to the remarkable clinical benefits of tyrosine-kinase inhibitors in NSCLC patients who harbor corresponding driver mutations, pretreatment multiplex genetic tests should be performed to guide therapeutic decisions. However, when tumor tissue is scarce, we may use clinicopathological features to predict specific genetic aberrations. Our study for the first time demonstrates that age at diagnosis alone can be a valuable tool to predict the presence of EML4-ALK rearrangement with fair sensitivity and specificity. While smoking pack-years but not age at diagnosis may predict EGFR mutations, as supported by previous and current study. However, the predictive power of smoking-pack-years reported here is less evident and we suggest not omitting heavier smokers from EGFR mutation testing in East Asian population.

Our study have several limitations. First, this is a single-institution study. However, we prospectively enrolled consecutive NSCLC

EML4-ALK $\approx$ EGFR $\approx$ WT/WT

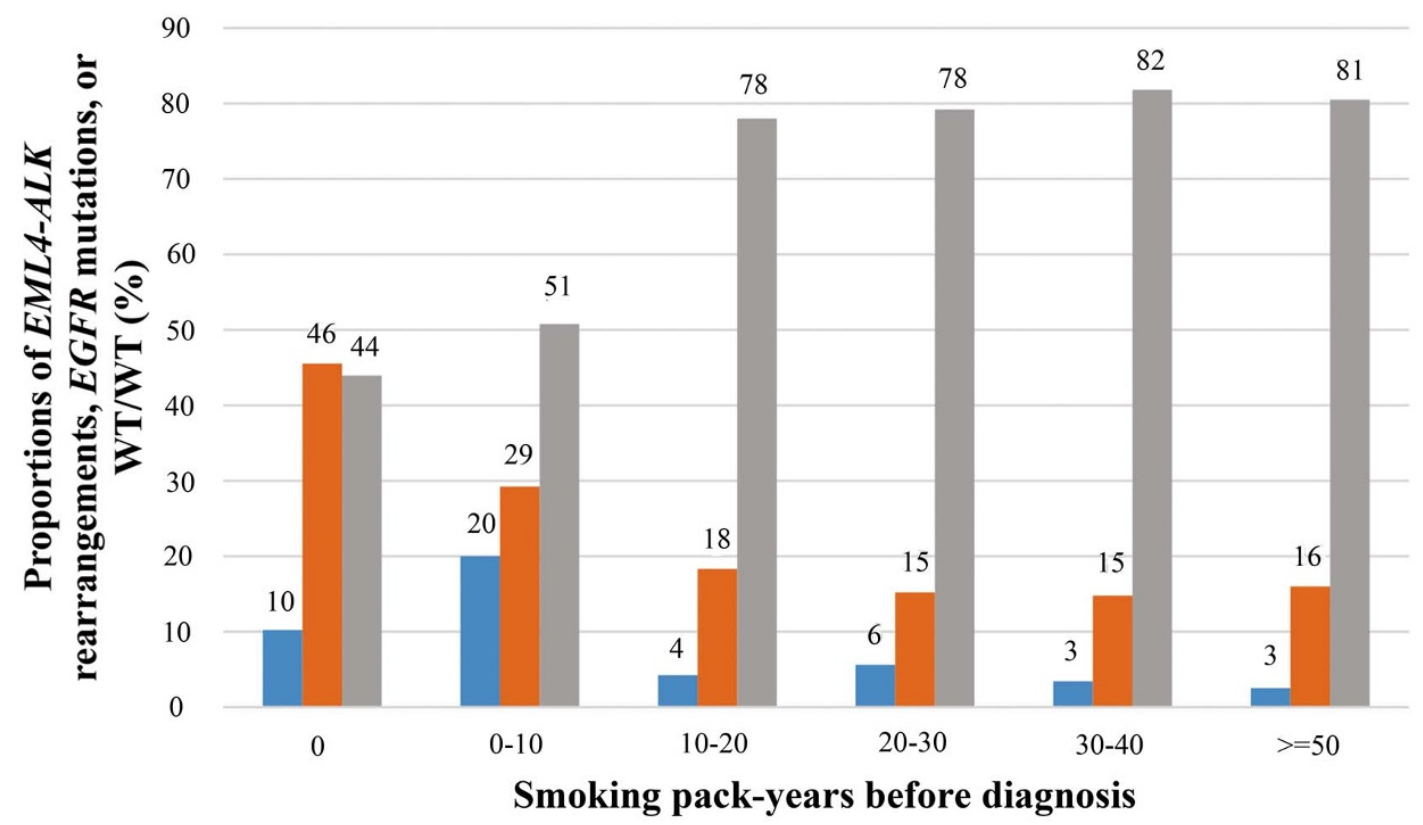

Figure $5 \mid$ The incidence of EML4-ALK rearrangements, EGFR mutations, and $W T / W T$ in non-small-cell lung cancer patients according to total smoking pack-years before diagnosis. WT/WT, wild type $A L K$ and EGFR. 


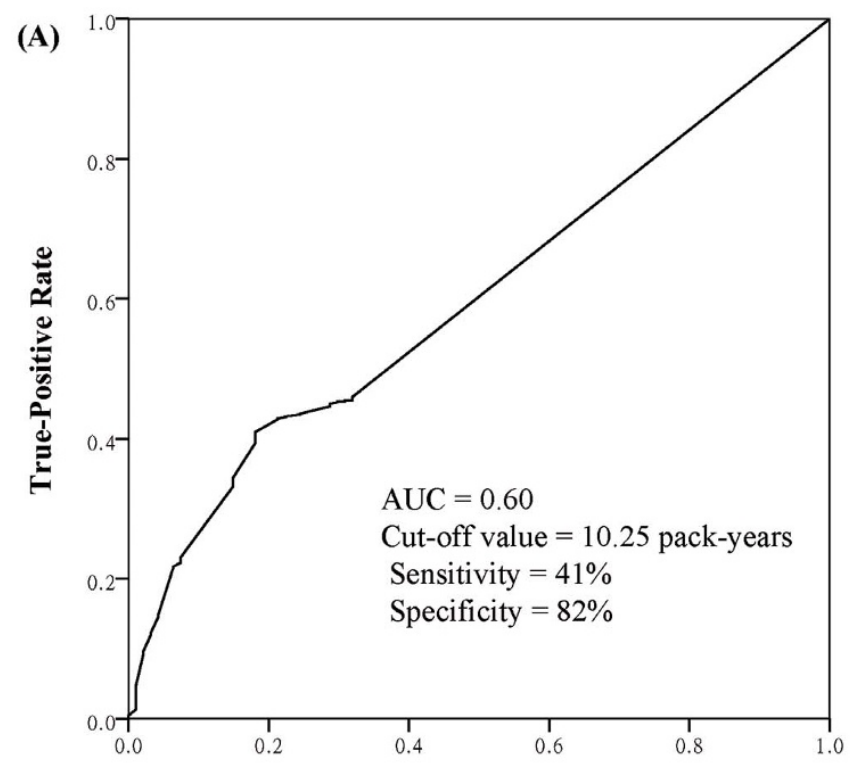

False-Positive Rate

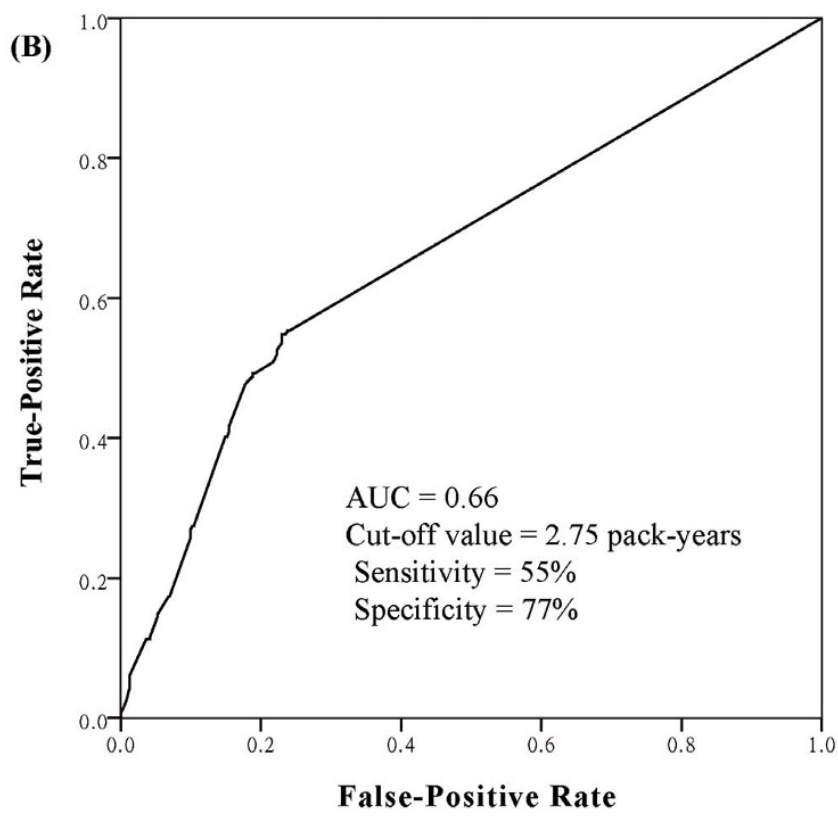

Figure 6 | Receiver operating characteristics (ROC) curves for total smoking pack-years before diagnosis as predictors of (A) EML4-ALK rearrangements and (B) EGFR mutations in non-small-cell lung cancer. AUC, areas under ROC curve.

patients seen in our hospital. We believe these unselected patients are fair representative of NSCLC patients across different pathological types. Second, we did not carry out survival analysis due to immature survival data. It would be interesting to evaluate the prognostic value of clinicopathological variables and mutation types. A recent study by $\mathrm{Li} \mathrm{C}$ et al. shows that no survival difference was noted in lung adenocarcinoma according to different driver mutations ${ }^{34}$. Yet, some studies found that ALK+ was associated with worse disease-free survival in NSCLC ${ }^{35,36}$. Overall, the prognostic value of different driver mutations are controversial which is probably due to more complicated treatment options in the era of targeted therapy. Third, we only focused on two currently druggable targets in NSCLC. Other driver mutations including KRAS, BRAF, HER2, MET, PTEN, RET, etc. have also been reported in previous studies ${ }^{35}$. Whether these driver share similar phenomenon needs further investigation.

In summary, we show that age at diagnosis alone is a valuable predictor of EML4-ALK rearrangements but poorly predicts EGFR mutations in NSCLC. Smoking pack-years may predict EGFR mutations though with limited power. We recommend the detection of EGFR mutations should not be confined to patients with "advantageous" features like younger patients, females and non-smoking. The results should help assess the likelihood of these two genetic aberrations based on available clinicopathological features and understand the biological implications of different driver mutations.

\section{Methods}

Patients and sample collection. This cross-sectional study was to determine the overall incidence of EML4-ALK rearrangement and EGFR mutation in Chinese Han population diagnosed as NSCLC; to investigate the distinct clinicopathological pathological features of patients who harbored EML4-ALK rearrangements or EGFR mutations; and to evaluate the predictive value of age at diagnosis and smoking packyears years for these two genetic aberrations. Patients who met the following criteria were prospectively enrolled: histologically or cytologically proven NSCLC patients by two independent pathologists (Y. Li and J.T. Jin); aged 18 years or older; able to provide informed consent; available and sufficient tumor tissue (biopsy or surgical specimen) for genomic analysis. Specimens were obtained from two sources: freshfrozen tumor samples from the Biobank of SYSUCC and formalin-fixed, paraffinembedded (FFPE) tissue submitted to the Department of Pathology (within 5 years before enrollment). The study was conducted in accordance with the Declaration of Helsinki, International Conference on Harmonisation Guidelines for Good Clinical Practice and was approved by the Ethics Committee of SYSUCC. Informed consent was obtained for each participant before the acquisition of tumor tissue.

Genetic analysis. EGFR mutations were detected using PCR-based direct sequencing of exons $18-21$ as previously described ${ }^{31}$. Briefly, genomic DNA was extracted from either tumors embedded in paraffin blocks or from fresh frozen tumors. PCR amplification was done using HotStarTaq DNA polymerase (Qiagen Inc., Valencia, CA) with a forward primer (5'-GGATCGGCCTCTTCATGC3') and a reverse primer (5'-TAAAATTGATTCCAATGCCATCC-3'). PCR products were sequenced directly using Applied Biosystems PRISM dye terminator cycle sequencing method (Perkin-Elmer Corp., Foster City, CA) with ABI PRISM 3100 Genetic Analyzer (Applied Biosystems, Foster City, CA). Any in-frame deletions in exon 19 or point mutations in exon 21 (L858R or L861Q substitutions), which confer sensitivity to EGFR-TKIs therapy, were considered as EGFR mutant. EML4-ALK rearrangements were detected by means of Fluorescence in situ Hybridization (FISH) using a breakapart probe to the ALK gene (Vysis LSI ALK Dual Color, Break Apart Rearrangement Probe; Abbott Molecular) per manufacturer's instructions. At least 100 representative tumor cells were counted. The results obtained by FISH were analyzed using an Olympus fluorescence microscope equipped with orange, green, and 4', 6-diamidino2-phenylindole filters. Images were captured using the Video Test Image Analysis System. FISH-positive cases were defined as $\geq 15 \%$ of the tumor cells that showed a split red and green signal and/or an isolated (single) red signal. Otherwise, the specimen was classified as ALK FISH negative.

Clinicopathological data. Clinicopathological features including age at diagnosis, gender, smoking history, pathological types, differentiation, cancer stage, symptoms at first onset and family history of malignant tumors were carefully collected. Lung cancer was histologically classified as adenocarcinoma, squamous cell carcinoma, adenosquamous carcinoma and other subtypes. Cancer stage was determined according to TNM classification according to the Union for International Cancer Control and the American Joint Committee on Cancer staging system, 7 th edition $^{32}$. Smokers were defined as those who had more than 100 lifetime cigarettes. Smoking pack-years was calculated as average number of cigarettes per day $/ 20 \times$ years smoking and was treated as a continuous variable.

Statistical analysis. Chi-square test (or Fisher exact test) and independent-samples ttest were applied to explore the univariate association between clinicopathological variables and specific genetic aberrations, for categorical and continuous data, respectively. All variables that were univariately associated at the level of $\alpha<0.2$ were included in the multivariate logistic regression model. Odds ratios (ORs) and 95\% confidence intervals (CIs) were calculated to denote the association. The receiver operating characteristic (ROC) curve methodology was applied to assess the ability of age at diagnosis or smoking pack-years to predict $E M L 4-A L K$ rearrangements and EGFR mutations. The diagnostic accuracy was presented as the area under the ROC curve (AUC). All statistical calculations were performed using SPSS version 21.0 (SPSS, Inc., Chicago, IL.) A two-tail $P$ value of $<0.05$ was judged significant.

1. Siegel, R., Ma, J., Zou, Z. \& Jemal, A. Cancer statistics, 2014. CA Cancer J Clin. 64, 9-29 (2014).

2. Mitsudomi, T. et al. Gefitinib versus cisplatin plus docetaxel in patients with nonsmall-cell lung cancer harbouring mutations of the epidermal growth factor 
receptor (WJTOG3405): an open label, randomised phase 3 trial. Lancet Oncol. 11, 121-128 (2010).

3. Rosell, R. et al. Erlotinib versus standard chemotherapy as first-line treatment for European patients with advanced EGFR mutation-positive non-small-cell lung cancer (EURTAC): a multicentre, open-label, randomised phase 3 trial. Lancet Oncol. 13, 239-246 (2012).

4. Wu, Y. L. et al. Afatinib versus cisplatin plus gemcitabine for first-line treatment of Asian patients with advanced non-small-cell lung cancer harbouring EGFR mutations (LUX-Lung 6): an open-label, randomised phase 3 trial. Lancet Oncol. 15, 213-222 (2014).

5. Cross, D. A. et al. AZD9291, an Irreversible EGFR TKI, Overcomes T790MMediated Resistance to EGFR Inhibitors in Lung Cancer. Cancer Discov. 4, 1046-1061 (2014)

6. Shaw, A. T. et al. Crizotinib versus chemotherapy in advanced ALK-positive lung cancer. N Engl J Med. 368, 2385-2394 (2013).

7. Shaw, A. T. et al. Ceritinib in ALK-rearranged non-small-cell lung cancer. NEnglJ Med. 370, 1189-1197 (2014).

8. Lynch, T. J. et al. Activating mutations in the epidermal growth factor receptor underlying responsiveness of non-small-cell lung cancer to gefitinib. $N$ Engl $J$ Med. 350, 2129-2139 (2004).

9. Pao, W., Iafrate, A. J. \& Su, Z. Genetically informed lung cancer medicine. J Pathol. 223, 230-240 (2011).

10. Shi, Y. et al. A prospective, molecular epidemiology study of EGFR mutations in Asian patients with advanced non-small-cell lung cancer of adenocarcinoma histology (PIONEER). J Thorac Oncol. 9, 154-162 (2014).

11. Pham, D. et al. Use of cigarette-smoking history to estimate the likelihood of mutations in epidermal growth factor receptor gene exons 19 and 21 in lung adenocarcinomas. J Clin Oncol. 24, 1700-1704 (2006).

12. Jida, M. et al. Usefulness of cumulative smoking dose for identifying the EGFR mutation and patients with non-small-cell lung cancer for gefitinib treatment. Cancer Sci. 100, 1931-1934 (2009).

13. Choi, Y. H. et al. Association between age at diagnosis and the presence of EGFR mutations in female patients with resected non-small cell lung cancer. $J$ Thorac Oncol. 5, 1949-1952 (2010).

14. Zhang, Y. et al. Frequency of driver mutations in lung adenocarcinoma from female never-smokers varies with histologic subtypes and age at diagnosis. Clin Cancer Res. 18, 1947-1953 (2012).

15. Ueno, T. et al. Impact of age on epidermal growth factor receptor mutation in lung cancer. Lung Cancer. 78, 207-211 (2012).

16. Soda, M. et al. Identification of the transforming EML4-ALK fusion gene in nonsmall-cell lung cancer. Nature 448, 561-566 (2007).

17. Soda, M. et al. A mouse model for EML4-ALK-positive lung cancer. Proc Natl Acad Sci U S A. 105, 19893-19897 (2008).

18. Kwak, E. L. et al. Anaplastic lymphoma kinase inhibition in non-small-cell lung cancer. N Engl J Med. 363, 1693-1703 (2010).

19. Camidge, D. R. et al. Activity and safety of crizotinib in patients with ALK-positive non-small-cell lung cancer: updated results from a phase 1 study. Lancet Oncol. 13, 1011-1019 (2012).

20. Zhang, X. et al. Fusion of EML4 and ALK is associated with development of lung adenocarcinomas lacking EGFR and KRAS mutations and is correlated with ALK expression. Mol Cancer. 9, 188 (2010).

21. Shaw, A. T. et al. Clinical features and outcome of patients with non-small-cell lung cancer who harbor EML4-ALK. J Clin Oncol. 27, 4247-4253 (2009).

22. Fallet, V. et al. Prospective screening for ALK: Clinical features and outcome according to ALK status. Eur J Cancer. 50, 1239-1246 (2014).

23. Rodig, S. J. et al. Unique clinicopathologic features characterize ALK-rearranged lung adenocarcinoma in the western population. Clin Cancer Res. 15, 5216-5223 (2009).

24. Koivunen, J. P. et al. EML4-ALK fusion gene and efficacy of an ALK kinase inhibitor in lung cancer. Clin Cancer Res. 14, 4275-4283 (2008).

25. Inamura, K. et al. EML4-ALK lung cancers are characterized by rare other mutations, a TTF-1 cell lineage, an acinar histology, and young onset. Mod Pathol. 22, 508-515 (2009).

26. Shinmura, K. et al. EML4-ALK fusion transcripts, but no NPM-, TPM3-, CLTC-, ATIC-, or TFG-ALK fusion transcripts, in non-small cell lung carcinomas. Lung Cancer. 61, 163-169 (2008).
27. Girard, N. et al. Nomogram to predict the presence of EGFR activating mutation in lung adenocarcinoma. Eur Respir J. 39, 366-372 (2012).

28. Tokumo, M. et al. The relationship between epidermal growth factor receptor mutations and clinicopathologic features in non-small cell lung cancers. Clin Cancer Res. 11, 1167-1173 (2005).

29. D'Angelo, S. P. et al. Incidence of EGFR exon 19 deletions and L858R in tumor specimens from men and cigarette smokers with lung adenocarcinomas. J Clin Oncol. 29, 2066-2070 (2011).

30. Bergethon, K. et al. ROS1 rearrangements define a unique molecular class of lung cancers. J Clin Oncol. 30, 863-870 (2012).

31. Wang, R. et al. RET fusions define a unique molecular and clinicopathologic subtype of non-small-cell lung cancer. J Clin Oncol. 30, 4352-4359 (2012).

32. Riely, G. J. et al. Frequency and distinctive spectrum of KRAS mutations in never smokers with lung adenocarcinoma. Clin Cancer Res. 14, 5731-5734 (2008).

33. Kawano, O. et al. PIK3CA mutation status in Japanese lung cancer patients. Lung Cancer. 54, 209-215 (2006).

34. Li, C. et al. Prognostic value analysis of mutational and clinicopathological factors in non-small cell lung cancer. PLoS One. 9, e107276 (2014).

35. Zhou, J. X. et al. Oncogenic driver mutations in patients with non-small-cell lung cancer at various clinical stages. Ann Oncol. 24, 1319-1325 (2013).

36. Yang, P. et al. Worse disease-free survival in never-smokers with ALK+ lung adenocarcinoma. J Thorac Oncol. 7, 90-97 (2012).

\section{Acknowledgments}

We thank all the patients who participated in this study and their families, the medical, nursing, and research staffs at the study center. We also thank Yong Li and Jietian Jin from the Department of Pathology in Sun Yat-sen University Cancer Center who reviewed all the specimens for pathological diagnosis. This work was supported by: Innovative drug R\&D center based on real-time high-throughput cell-based screening platform and large capacity compound library (Grant No: 2013ZX09401003-002), National Natural Science Funds of China (Grant No: 81372502) and National High Technology Research and Development Program of China (Grant No: 2012AA02A502), Wu Jieping Medical Foundation Project (Grant No: 08-JC-003).

\section{Author contributions}

S.H. and L.Z. conceived and designed the study; S.H., W.F., Z.H., T.Z. and Y.Y. performed the study; S.H., W.F., Y.T., Y.M., Y.Z. and C.X. analyzed the data; all the authors (S.H., W.F., Z.H., T.Z., Y.Y., T.Q., Y.T., Y.M., Y.Z., C.X., Y.H., H.Z. and L.Z.) wrote the article. All authors (S.H., W.F., Z.H., T.Z., Y.Y., T.Q., Y.T., Y.M., Y.Z., C.X., Y.H., H.Z. and L.Z.) have read and approved the final manuscript.

\section{Additional information}

Supplementary information accompanies this paper at http://www.nature.com/ scientificreports

Competing financial interests: Yes there is potential Competing Interest. Li Zhang has received research support from Boehringer Ingelheim, Astra Zeneca, Lilly, and Roche. Other authors declare no conflicts of interest.

How to cite this article: Hong, S. et al. A Large-scale Cross-sectional Study of ALK Rearrangements and EGFR Mutations in Non-small-cell Lung Cancer in Chinese Han Population. Sci. Rep. 4, 7268; DOI:10.1038/srep07268 (2014).

This work is licensed under a Creative Commons Attribution-NonCommercialNoDerivs 4.0 International License. The images or other third party material in this article are included in the article's Creative Commons license, unless indicated otherwise in the credit line; if the material is not included under the Creative Commons license, users will need to obtain permission from the license holder in order to reproduce the material. To view a copy of this license, visit http:// creativecommons.org/licenses/by-nc-nd/4.0/ 